\title{
BAsE-Seq: a method for obtaining long viral haplotypes from short sequence reads
}

\author{
Lewis Z Hong ${ }^{1,8}$, Shuzhen Hong ${ }^{1,9}$, Han Teng Wong ${ }^{1}$, Pauline PK Aw², Yan Cheng ${ }^{3}$, Andreas Wilm², \\ Paola F de Sessions' ${ }^{2}$ Seng Gee Lim ${ }^{3,4}$, Niranjan Nagarajan ${ }^{2}$, Martin L Hibberd ${ }^{2,5}$, Stephen R Quake ${ }^{6,7^{*}}$ \\ and William F Burkholder ${ }^{1 *}$
}

\begin{abstract}
We present a method for obtaining long haplotypes, of over $3 \mathrm{~kb}$ in length, using a short-read sequencer, Barcode-directed Assembly for Extra-long Sequences (BAsE-Seq). BAsE-Seq relies on transposing a template-specific barcode onto random segments of the template molecule and assembling the barcoded short reads into complete haplotypes. We applied BAsE-Seq on mixed clones of hepatitis B virus and accurately identified haplotypes occurring at frequencies greater than or equal to $0.4 \%$, with $>99.9 \%$ specificity. Applying BAsE-Seq to a clinical sample, we obtained over 9,000 viral haplotypes, which provided an unprecedented view of hepatitis B virus population structure during chronic infection. BAsE-Seq is readily applicable for monitoring quasispecies evolution in viral diseases.
\end{abstract}

\section{Background}

The ability of viruses to escape host immune responses or develop drug resistance represents a significant challenge to human health. Successful viral evolution is driven by high mutation rates that generate genetically diverse populations within an infected host, which are referred to as viral quasispecies $[1,2]$. Genetic interactions between mutant viruses within a quasispecies have been proposed to affect the overall fitness of the population through a combination of cooperative and antagonistic effects [3-6]. In recent years, next-generation DNA sequencing technologies have been used to perform ultra-deep sequencing of bulk samples to detect signatures of viral quasispecies by measuring allele distributions of single nucleotide variants (SNVs) [7-10] (this approach is hereafter referred to as 'Deep-Seq'). This approach was recently refined through the development of methods based on redundant sequencing of barcode-tagged or circularized template molecules to reduce the error rates associated with next-generation sequencing, enabling the detection of lower frequency SNVs [11-13]. However, any meaningful attempt to study intra-quasispecies interactions will require the ability to

\footnotetext{
* Correspondence: quake@stanford.edu; wfburkholder@gmail.com ${ }^{6}$ Departments of Bioengineering and Applied Physics, Stanford University and Howard Hughes Medical Institute, Stanford, CA 94305, USA 'Institute of Molecular and Cell Biology, Agency for Science, Technology and Research, Singapore 138673, Singapore

Full list of author information is available at the end of the article
}

determine viral haplotypes (here, 'haplotype' refers to the set of SNVs that occur on a particular copy of the viral genome) so that the correlation and co-occurrence of SNVs within quasispecies can be characterized. Unfortunately, most sequencing platforms are inherently inadequate with respect to resolving haplotype information beyond several hundred base pairs due to limitations on read length [14], and existing assembly algorithms for haplotype reconstruction from quasispecies suffer from poor sensitivity and specificity [15].

There are several possible approaches for determining haplotypes of viruses with long genomes ('long-range haplotypes'; >1 kb) using existing sequencing technology. One possibility is to use a long-read single-molecule sequencing platform such as the PacBio RS II or nanoporebased sequencers. However, the high intrinsic error rate of the PacBio platform necessitates redundant sequencing across the same template to obtain an accurate consensus sequence, thereby substantially decreasing the effective read length of the technology [16,17]. Sequencers based on nanopore technology are still in development; the accuracy and scalability of this type of technology are currently unclear $[18,19]$. Another possibility for obtaining long-range haplotypes is to extend the effective singlemolecule read length obtained from short-read platforms. To that end, several methods have been developed. A method developed by Hiatt et al. [20] relied on tagging 
individual DNA molecules with a unique sequence, followed by paired-end sequencing of nested breakpoints and performing hierarchical local assembly to reconstruct the template sequence. This method produced 'subassemblies' that were only approximately $700 \mathrm{bp}$ in size due to constraints in the size of molecules that can be efficiently processed on the sequencer. Schwartz et al. [21] developed a method that involved stretching long DNA molecules on an Illumina flow cell, followed by in situ library construction. Sequence reads that originated from the same molecule were combined by relying on spatial information from the flow cell. This method is technically challenging as it involves customized modification of the sequencer. Single virion sequences have been obtained by molecular cloning or by serial dilution of cDNA or DNA molecules to achieve $\leq 1$ copy per reaction, followed by a 'primer walking' method using capillary sequencing to obtain clonal sequences [22-26]. In principle, this approach can produce high quality haplotypes where sequence length will only be limited by technical constraints in performing molecular cloning and long-range PCR, but suffers from relatively low throughput due to high cost for reagents and labor. Several methods have been developed that rely on physical separation of DNA molecules into reaction chambers containing one or few molecules using limiting dilution or microfluidics, and assembling haplotypes from short reads generated by performing nextgeneration sequencing on individual reaction chambers [27-30]. Using this approach requires independent library preparation from each reaction chamber, thereby limiting the number of haplotypes that can be obtained per experiment.

Here, we report a method that obtains long haplotypes (>3 kilobases) from viral samples using a short-read sequencer: BAsE-Seq or Barcode-directed Assembly for Extra-long Sequences. BAsE-Seq takes advantage of the low cost-per-base and low error rates of short-read sequencing platforms and addresses the limitations of current methods for extending read lengths. BAsE-Seq relies on attaching unique molecular barcodes to long template molecules, followed by transposing the barcode to random overlapping segments of the template; barcode-tagged sequence reads derived from the same template molecules can be combined to obtain highly accurate haplotype sequences. BAsE-Seq was used to perform single virion sequencing of hepatitis B virus (HBV), which has an approximately $3.2 \mathrm{~kb}$ genome and exists as a quasispecies within its host [31-33]. We first describe using BAsE-Seq to obtain individual genome sequences at high accuracy from mixed samples of HBV clones and demonstrate the assembly of viral haplotypes at high sensitivity and specificity. Next, we used BAsE-Seq to obtain the first-time measurement of $>9,000$ viral haplotypes from a clinical sample. Our method showed good agreement in SNV and haplotype identification when compared with Deep-Seq and clonal sequencing, and allowed us to investigate intra-host phylogenetic structure of HBV quasispecies during chronic infection.

\section{Results and discussion \\ Overview of BAsE-Seq}

An outline of the BAsE-Seq methodology is shown in Figure 1a. The basic workflow involves attaching unique barcodes to full-length HBV genomes, and then constructing a library where the barcode is juxtaposed to random overlapping fragments of its assigned genome. Barcode assignment is performed using a pair of primers that contain HBV-specific sequences on their $3^{\prime}$ ends and universal sequences on their $5^{\prime}$ ends (Figure S1 in Additional file 1). Subsequently, barcode-tagged genomes are clonally amplified by PCR using universal primers and exonuclease-mediated digestion is initiated from the barcode-distal end to obtain a broad size distribution of barcode-containing fragments. Next, these fragments are circularized by intramolecular ligation, which juxtaposes different regions of the viral genome adjacent to its assigned barcode. The circularized molecules are used as a template for random fragmentation and adapter tagging using Nextera transposomes, followed by PCR enrichment of the sequencing library to incorporate Illumina-specific paired-end adapters and enrich for barcode-tagged molecules. The library is loaded on a MiSeq for $2 \times 150 \mathrm{bp}$ sequencing and a custom sequencing primer is used for the second read to obtain the barcode sequence.

After sequencing, barcode-containing read pairs are used to generate a 'bulk consensus' genome by iterative alignment of read pairs against a HBV reference sequence from GenBank. Next, aligned read pairs are de-multiplexed based on barcode identity, and 'individual consensus' genomes are obtained by extracting the consensus base call at each position. Finally, SNVs relative to the bulk consensus genome are identified from individual genomes and used to construct haplotypes (Figure 1b).

\section{Developing BAsE-Seq for single virion sequencing}

In this subsection, we will discuss the critical steps of our protocol and several challenges that were overcome during the development of BAsE-Seq.

The forward primer used during barcode assignment contains a string of 20 random nucleotides, which allows for approximately $1.1 \times 10^{12}$ possible barcode sequences. Barcode assignment is performed using two cycles of PCR on a template containing $10^{6}$ double-stranded HBV genomes. This represents an approximately 55,000-fold excess of possible barcode sequences to template molecules; each strand of the genome will be uniquely tagged by a barcode and flanked by universal sequences (Figure S1 in Additional file 1). With this approach, random errors 


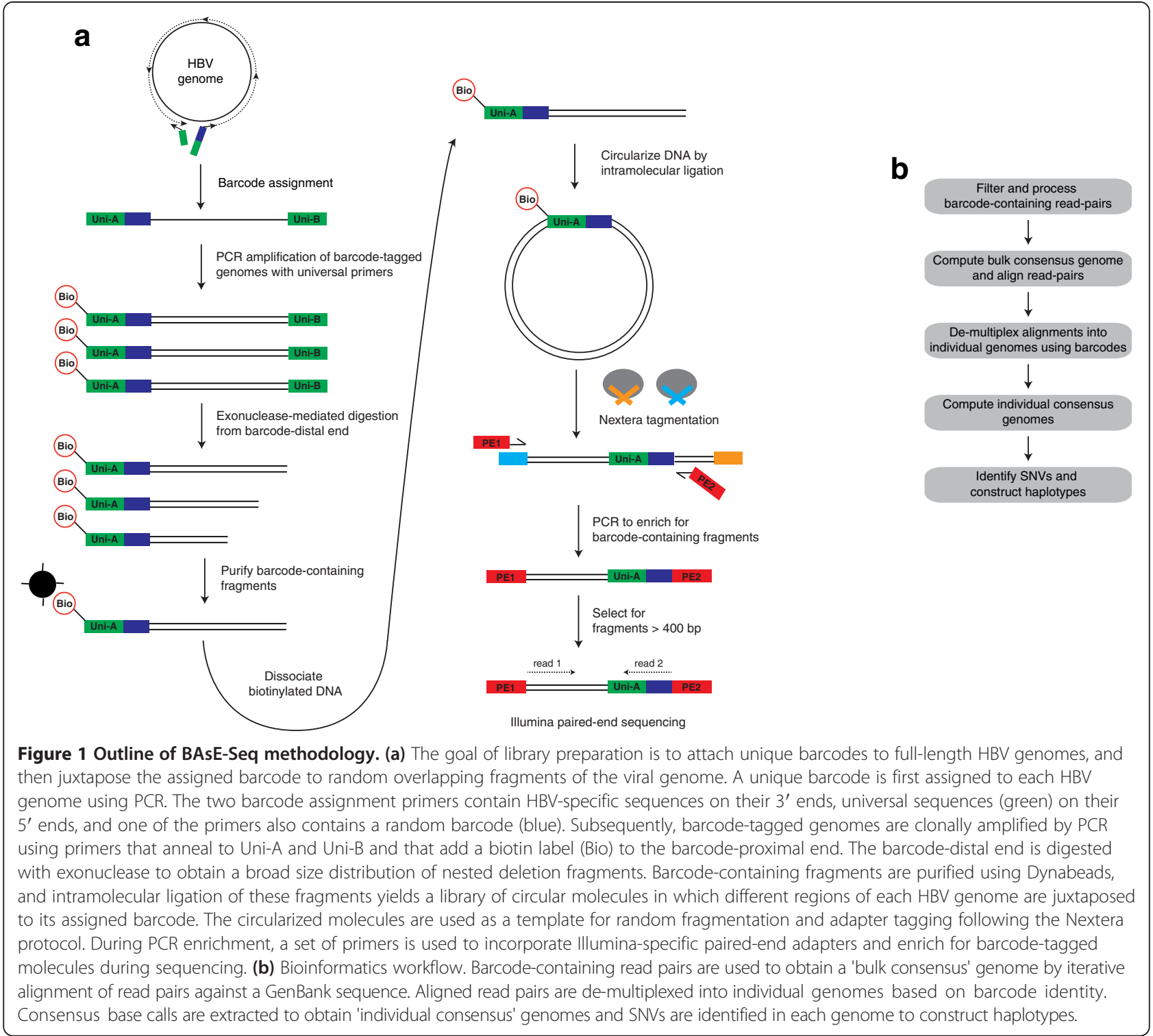

that are introduced subsequent to barcode assignment, such as during library preparation or sequencing, can be removed (Figure S1 in Additional file 1) [13,34]. Hence, the only errors that will remain are PCR errors that were incorporated during barcode assignment or systematic errors that occurred during library preparation or sequencing.

There are two steps in BAsE-Seq that are critical for producing uniform genome coverage: (a) generating deletions from the barcode-distal end of each HBV genome and (b) generating a sequencing library from circularized molecules. In the former, the goal is to obtain an equimolar size distribution of barcode-tagged HBV genomes containing nested deletions from the barcode-distal end, as it will expose different regions along the genome for juxtaposition with the barcode during circularization.
This was achieved using a classical approach in which exonuclease III was used for processive digestion from an unprotected end (barcode-distal end) of the template and aliquots were removed at defined time intervals for S1 nuclease digestion to create blunt ends [35]. In the latter, an efficient method was required to fragment circularized molecules and attach sequencing adaptors onto barcode-containing fragments. To achieve this, we relied on a transposase-catalyzed method (Illumina) that is known to introduce slightly higher bias in fragmentation compared with conventional methods, but offered significant advantages in its simplicity and ability to handle low input material [36].

To generate a suitable HBV template for protocol development, we isolated two different HBV clones that contained 17 single nucleotide polymorphisms (SNPs) between 
them - hereafter referred to as Clone- 1 and Clone-2 (Tables S1 and S2 in Additional file 1). During the initial phase of protocol development, we used a 1:1 mixture of Clone-1 and Clone- 2 for library preparation in order to assess the ability of our protocol to generate accurate haplotype sequences. Subsequent analysis of sequence data that were generated from the first round of libraries showed that the majority of haplotypes were chimeric, i.e., contained SNPs from both Clone-1 and Clone-2 (data not shown). To identify the steps in our protocol where molecular chimerism was occurring, we prepared libraries in which samples derived from Clone-1 and Clone- 2 were mixed at different steps along the protocol. Using this approach, we determined that chimeric sequences were mostly generated during (a) the PCR amplification step that occurred immediately after barcode assignment and (b) the circularization step. Taking reference from previous studies where it was demonstrated that PCR-induced chimeras could be reduced by limiting the number of PCR cycles [37,38], we developed a real-time PCR assay to monitor the PCR efficiency at this step and realized that PCRs that were stopped during the log-linear phase of amplification produced significantly less chimeric sequences. This led us to develop a twostage PCR protocol to amplify barcode-tagged HBV genomes (further described in Appendix B in Additional file 2) that minimized the formation of PCR-induced chimeras and provided enough PCR products to continue with library preparation. To identify reaction conditions for double-stranded DNA circularization that maximized intramolecular ligation and minimized intermolecular ligation (which will result in the formation of chimeric sequences), we mixed two sub-genomic HBV sequences each approximately $1 \mathrm{~kb}$ long - at equimolar amounts and used them as template for circularization. We developed a quantitative PCR assay (further described in Appendix C in Additional file 2) to measure the abundance of junctions formed by inter- or intra-molecular ligation. This assay allowed us to screen a large number of reaction conditions and identified two key parameters that were critical for optimal circularization: reaction volume and temperature. Notably, a significantly higher rate of intramolecular ligation (approximately $5 \%$ ) was achieved by increasing the reaction volume to $45 \mathrm{ml}$ and decreasing the reaction temperature to $10^{\circ} \mathrm{C}$. Ultimately, a combination of optimized conditions at both steps - PCR amplification and circularization - allowed us to produce haplotype sequences with minimal chimerism (as presented below).

\section{Validation of BAsE-Seq with mixed hepatitis B virus clones}

To assess the accuracy and sensitivity of BAsE-Seq in performing single virion sequencing on $\mathrm{HBV}$, we mixed Clone-1 and Clone-2 at unequal ratios (1:9 and 1:99) prior to barcode assignment and library preparation, yielding two BAsE-Seq libraries: Lib_1:9 and Lib_1:99. Each library was sequenced on a single run on the MiSeq, producing 6 to 8 million read pairs that could be aligned concordantly to the bulk consensus genome (Table 1; Figure S2 in Additional file 1). Subsequently, each library was analyzed using the 'bulk' approach or the 'individual' genome approach. In the bulk analysis, barcode information was ignored, i.e., sequence reads were not de-multiplexed, and the aligned read pairs were analyzed using a typical pipeline for Deep-Seq in which BAM files were used as input for variant calling using LoFreq [39,40]. In the individual genome analysis, aligned read pairs associated with unique barcodes were analyzed separately as described earlier (Figure 1b).

At the individual genome level, average per-base coverage was high ( $>50$ unique reads) for both libraries across the majority of bases in the genome (Figure S3 and Table S3 in Additional file 1). We obtained 2,390 and 3,673 high quality genomes - $\geq 4$ unique reads per base position across $\geq 85 \%$ of the genome - from Lib_1:9 and Lib_1:99, respectively (Table 1). These high quality genomes were used in downstream analysis for SNV identification, error analysis, and haplotype analysis.

The bulk analysis identified all 17 true SNVs at an average minor allele frequency (MAF) of $14.3 \pm 1.9 \%$ in Lib_1:9 and 15 out of 17 true SNVs at an average MAF of $0.712 \pm 0.264 \%$ in Lib_1:99. In comparison, the individual genome analysis identified all 17 true SNVs in both libraries, at an average MAF of $11.4 \pm 0.2 \%$ in Lib_1:9 and 0.394 $\pm 0.026 \%$ in Lib_1:99 (Figure 2). Since the true consensus sequence is known and the error rate of plasmid replication is extremely low (approximately $5 \times 10^{-10}$ ) [41], variants at non-SNP positions can be classified as errors generated by our protocol; the overall error rate was approximately seven-fold lower in the individual genome analysis (Table 1). Furthermore, the highest per-base error rate is approximately $0.2 \%$ in the individual genome analysis, which is approximately nine-fold lower compared with the bulk analysis (Table 1). These results indicate that our consensus base-calling approach using barcodes can substantially reduce the error rate of nextgeneration sequencing, thereby increasing the sensitivity and specificity of detecting SNVs occurring at $\leq 2 \%$ frequency (Table 1 and Figure 2). Further, using barcodes to tag individual genomes provides more precise estimation of SNV frequencies, as was evident in lower standard deviations in SNV frequencies observed in the individual genome analysis.

Based on the fidelity of the Long PCR Enzyme Mix reported by the manufacturer $\left(7.3 \times 10^{-6}\right.$ errors per nucleotide per PCR cycle), the expected error rate during barcode assignment is approximately $0.0015 \%$ - approximately one error in $22 \mathrm{HBV}$ genomes - which sets the expected error rate for BAsE-Seq. However, the error rate for both Lib_1:9 and Lib_1:99 is approximately four-fold higher (Table 1). 
Table 1 Summary statistics from BAsE-Seq and Deep-Seq of hepatitis B virus

\begin{tabular}{|c|c|c|c|c|c|c|c|}
\hline \multirow[b]{2}{*}{ Read-pairs } & \multicolumn{2}{|c|}{ Lib_1:9 } & \multicolumn{2}{|c|}{ Lib_1:99 } & \multicolumn{3}{|c|}{ S7.1 } \\
\hline & \multicolumn{2}{|c|}{$14,352,128$} & \multicolumn{2}{|c|}{$17,083,497$} & \multicolumn{2}{|c|}{$42,997,995$} & $8,197,770$ \\
\hline Library & \multicolumn{2}{|c|}{ BAsE-Seq } & \multicolumn{2}{|c|}{ BAsE-Seq } & \multicolumn{2}{|c|}{ BAsE-Seq } & Deep-Seq \\
\hline Type of sample & \multicolumn{2}{|c|}{ Mixed clone } & \multicolumn{2}{|c|}{ Mixed clone } & Internal standard & Patient & Patient \\
\hline Pass-filter read pairs ${ }^{\mathrm{a}}$ & \multicolumn{2}{|c|}{$6,751,411(47 \%)$} & \multicolumn{2}{|c|}{$8,816,934(52 \%)$} & $545,960(1 \%)$ & $26,066,408(61 \%)$ & $6,351,796(77 \%)$ \\
\hline Concordantly aligned $^{\mathrm{b}}$ & \multicolumn{2}{|c|}{$6,027,421(89 \%)$} & \multicolumn{2}{|c|}{$8,150,721(92 \%)$} & $496,356(91 \%)$ & $23,366,358(90 \%)$ & $4,261,572(67 \%)$ \\
\hline High quality genomes & \multicolumn{2}{|c|}{$2,390^{c}$} & \multicolumn{2}{|c|}{$3,673^{c}$} & $345^{d}$ & $12,444^{\mathrm{d}}$ & \\
\hline Type of analysis & Bulk & Individual & Bulk & Individual & Individual & Individual & \\
\hline Median per-base coverage depth & 333,677 & 86 & 470,036 & 63 & 38 & 45 & 131,492 \\
\hline True SNVs detected & $17 / 17$ & $17 / 17$ & $15 / 17$ & $17 / 17$ & & 68 & \\
\hline SNVs detected & & & & & & & 308 \\
\hline Errors detected & 522 & 218 & 328 & 257 & 11 & & \\
\hline Highest per-base error & $1.91 \%$ & $0.202 \%$ & $2.14 \%$ & $0.231 \%$ & $0.69 \%$ & & \\
\hline Overall error & $0.0524 \%$ & $0.00674 \%$ & $0.0324 \%$ & $0.00541 \%$ & $0.00214 \%$ & & \\
\hline
\end{tabular}

Each BAsE-Seq library was analyzed using the 'bulk' approach, i.e., without de-multiplexing by barcode identity, or the 'individual' approach, i.e., sequence reads associated with unique barcodes were analyzed separately. True SNVs in S7.1 were identified by BAsE-Seq as those that occurred at $>0.69 \%$ frequency. ${ }^{a}$ Read pairs after removal of adaptor and/or universal sequences. For BAsE-Seq libraries, this only includes read pairs that carry a barcode.

${ }^{b}$ Both reads in a pair were aligned in the expected orientation.

$c^{c} \geq 4$ unique reads per base position across $\geq 85 \%$ of the genome.

${ }^{d} \geq 4$ unique reads per base position across $\geq 50 \%$ of the genome.

Interestingly, the errors from individual genomes in both libraries have a significant overlap with regards to base position $(P<4 \mathrm{e}-14$, Fisher's exact test), which suggests that some of the errors were not removed by our approach because they were introduced systematically. It is also likely that the higher than expected error rate could be because the PCR polymerase used during barcode assignment has a higher error rate than is reported by the manufacturer.

For both Lib_1:9 and Lib_1:99, the observed frequencies of the Clone-1 and Clone-2 haplotypes were very close to the expected frequencies (Table 2). In addition to the expected haplotype sequences, two haplotype sequences were detected in each library that differed from the sequences of the two clones used for library preparation. These haplotypes could be the result of molecular chimeras that formed during library preparation. Alternatively, given their low frequency $(\leq 0.05 \%)$ and the presence of only one discordant SNV in each haplotype sequence, they could be the result of errors in individual genome sequences. Taken together, these results indicate that BAsE-Seq generates highly sensitive and accurate SNV calls and haplotypes from viral samples.

\section{Evaluation of BAsE-Seq on a patient sample}

To evaluate the performance of BAsE-Seq on a clinical sample, BAsE-Seq and Deep-Seq libraries were generated using viral DNA isolated from a chronic hepatitis B patient. This patient sample is hereafter referred to as 'S7.1'. Sequence reads from the Deep-Seq library were used to generate a bulk consensus genome for S7.1, and subsequent alignment to this bulk consensus genome produced a median per-base coverage depth of 131,492 reads (Table 1; Figure S4 in Additional file 1). We identified 308 SNVs from the Deep-Seq library, ranging in frequency from $0.2 \%$ to $47 \%$ (Table 1 and Figure 2e). In the BAsESeq library, we used an internal standard in order to estimate error frequencies during library preparation and sequencing; the internal standard acts as a control for errors observed in the library that it was prepared from. We prepared the internal standard by assigning barcodes separately to HBV Clone-2; these barcodes contained a two-base insertion that allowed us to distinguish them from patient-specific viral genomes. After barcode assignment, the internal standard was mixed with patientspecific viral genomes and used to build a BAsE-Seq library. Among 345 high quality genomes derived from the internal standard, the highest per-base error rate was $0.69 \%$, which we termed the 'baseline error frequency' for the library. Using the baseline error frequency as a threshold below which a SNV might be due to an error and above which a SNV was treated as a true SNV, we identified 68 true SNVs among the 12,444 high quality genomes assembled from S7.1 (Table 1). The SNVs were evenly distributed across the genome and had a large frequency range: 17 SNVs occurred below $1 \%$ frequency, 24 SNVs occurred between $1 \%$ and 10\% frequency, and 27 SNVs occurred above 10\% frequency (Figure 2f). Although our pipeline should preclude SNVs occurring above $50 \%$ frequency, one SNV was identified by BAsE-Seq at approximately $62 \%$ frequency because the bulk consensus genome was generated using sequence reads from 

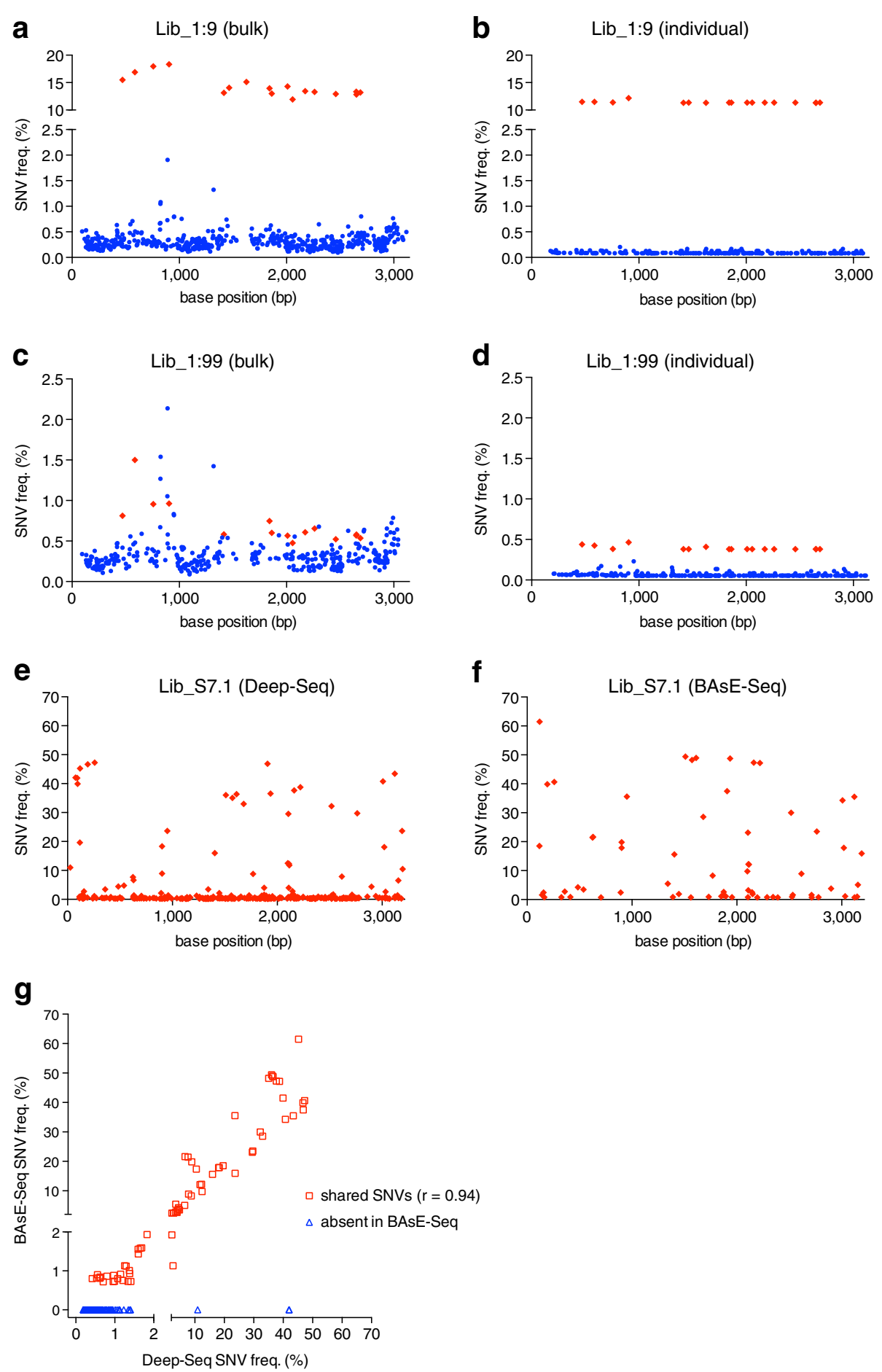

Figure 2 SNVs in BAsE-Seq and Deep-Seq libraries. (a-d) SNVs in BAsE-Seq libraries Lib_1:9 and Lib_1:99 were identified as true SNVs (red diamonds) or errors (blue dots) using the 'bulk' approach $(\mathbf{a}, \mathbf{c})$ or the 'individual' approach $(\mathbf{b}, \mathbf{d})$. The frequency of each SNV (y-axis) is plotted against base position in the consensus sequence (x-axis). Additional information is also provided in Tables 1 and 3. (e,f) SNVs from S7.1 were identified using Deep-Seq and BAsE-Seq. The BAsE-Seq library contained an internal standard that was used to calculate the error-free frequency cutoff for the library; hence, only error-free SNVs are shown in the BAsE-Seq analysis of S7.1. (g) The frequency of SNVs detected in the BAsE-Seq library (y-axis) is plotted against the frequency of SNVs detected in the Deep-Seq library ( $\mathrm{x}$-axis). All 68 error-free SNVs identified by BAsE-Seq were also identified by Deep-Seq (Pearson correlation coefficient $=0.94$ ). 
Table 2 Haplotypes identified by BAsE-Seq in Lib_1:9 and Lib_1:99

\begin{tabular}{|c|c|c|c|c|c|c|}
\hline \multirow{3}{*}{ Haplotypes $^{a}$} & \multicolumn{3}{|c|}{ Lib_1:9 } & \multicolumn{3}{|c|}{ Lib_1:99 } \\
\hline & \multicolumn{2}{|c|}{ Individual $^{\mathbf{b}}$} & \multirow{2}{*}{$\frac{\text { Bulk }^{\mathbf{c}}}{85.7 \%}$} & \multicolumn{2}{|c|}{ Individual $^{\mathbf{b}}$} & \multirow{2}{*}{$\begin{array}{l}\text { Bulk } \\
99.3 \%\end{array}$} \\
\hline & 2,118 & $88.6 \%$ & & 3,656 & $99.5 \%$ & \\
\hline ITTAAACAG & 270 & $11.3 \%$ & $14.3 \%$ & 14 & $0.4 \%$ & $0.7 \%$ \\
\hline$\sigma$ & 1 & $0.04 \%$ & $0 \%$ & 1 & $0.03 \%$ & $0 \%$ \\
\hline ACACTAAATTTAAA...AG & 1 & $0.04 \%$ & $0 \%$ & 0 & $0 \%$ & $0 \%$ \\
\hline$\Delta$ & 0 & $0 \%$ & $0 \%$ & 2 & $0.05 \%$ & $0 \%$ \\
\hline otal & 2,390 & $100 \%$ & $100 \%$ & 3,673 & $100 \%$ & $100 \%$ \\
\hline
\end{tabular}

${ }^{\mathrm{a}}$ Haplotypes observed in individual genomes. The major allele (Clone-2) is represented by a period and the minor allele (Clone-1) is represented by the base at that position (Table S2 in Additional file 2).

${ }^{\mathrm{b}}$ Haplotype frequencies observed in individual genomes.

'Average allele frequency of SNPs from 'bulk' analysis.

Deep-Seq, where this variant was found to occur at a frequency just below $50 \%$.

There was good agreement between BAsE-Seq and Deep-Seq in SNV identification: all 68 true SNVs identified by BAsE-Seq were also found by Deep-Seq and SNV frequencies were highly correlated between both methods (Figure $2 \mathrm{~g}$ ). Five SNVs at $>10 \%$ frequency were detected by Deep-Seq but were missed by BAsE-Seq because these SNVs were all located within $60 \mathrm{bp}$ of the BAsE-Seq primer binding sites, where per-base coverage using BAsESeq was significantly lower (Figure $2 \mathrm{~g}$ ). All of the remaining
SNVs that were only detected by Deep-Seq occurred at frequencies $<1.4 \%$ (Figure 2g). Among these SNVs, 217 were also found in the BAsE-Seq data but occurred below the baseline error frequency used as a cutoff; it is unclear whether these are true SNVs or errors. The remaining 18 SNVs were not found in the BAsE-Seq data despite good per-base coverage, and are likely to be errors specific to Deep-Seq.

To validate the accuracy of haplotypes observed by BAsE-Seq in S7.1, 20 sub-genomic clones containing the pre-core and basal core promoter region were isolated. Capillary sequencing was performed, which detected a total of five SNVs and five unique haplotypes across a $367 \mathrm{bp}$ region (Table 3). In total, BAsE-Seq analysis of S7.1 identified 2,555 haplotypes with a 100\% SNV calling rate across this region. Ten unique haplotypes were detected; four of these haplotypes were also observed in the sequenced clones, which includes the three most frequently observed haplotypes and a haplotype that was detected at approximately $0.08 \%$ frequency in BAsE-Seq (Table 3). These results indicate that BAsE-Seq is capable of highly sensitive, accurate and quantitative identification of single virion sequences from a clinical sample.

\section{Analysis of haplotypes in the patient sample}

Of the 68 SNVs identified by BAsE-Seq in S7.1, 56 confer changes in amino acid sequence, while the other 12 are

Table 3 Comparison of haplotypes observed over a 367 bp region in S7.1

\begin{tabular}{|c|c|c|c|c|c|c|c|}
\hline SNV position ${ }^{\mathrm{a}}$ & 118 & 119 & 192 & 258 & 484 & & \\
\hline Consensus base $^{\mathbf{b}}$ & $T$ & G & C & G & $\mathrm{C}$ & & \\
\hline Variant base & G & A & $T$ & $\mathrm{C}$ & A & & \\
\hline Deep-Seq frequency (\%) & 19.6 & 45.2 & 46.7 & 47.3 & 4.5 & & \\
\hline \multirow[t]{7}{*}{ BAsE-Seq frequency (\%) } & 18.5 & 61.5 & 39.9 & 40.6 & 4.2 & & \\
\hline & & & & & & Observed in clones & Observed in BAsE-Seq \\
\hline & & A & & & . & $10(50 \%)$ & $1,588(62 \%)$ \\
\hline & . & & $\mathrm{T}$ & C & . & $2(10 \%)$ & $428(17 \%)$ \\
\hline & G & . & $\mathrm{T}$ & C & & $5(25 \%)$ & $403(16 \%)$ \\
\hline & . & . & $\mathrm{T}$ & C & A & 0 & $65(3 \%)$ \\
\hline & G & . & $\mathrm{T}$ & C & A & 0 & $27(1 \%)$ \\
\hline \multirow[t]{6}{*}{ Haplotypes ${ }^{c}$} & . & . & . & . & & 0 & $24(1 \%)$ \\
\hline & G & A & $\mathrm{T}$ & C & . & 0 & $14(0.5 \%)$ \\
\hline & 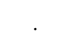 & A & & . & A & $1(5 \%)$ & $2(0.08 \%)$ \\
\hline & . & . & $\mathrm{T}$ & . & & 0 & $2(0.08 \%)$ \\
\hline & G & & & C & . & 0 & $2(0.08 \%)$ \\
\hline & & A & $\mathrm{T}$ & C & . & $2(10 \%)$ & 0 \\
\hline Unique haplotypes & & & & & & 5 & 10 \\
\hline Total observed haplotypes & & & & & & 20 & 2,555 \\
\hline
\end{tabular}

${ }^{a}$ SNVs identified by all three methods - Sanger sequencing of clones, Deep-Seq and BAsE-Seq.

${ }^{b}$ Base call in the bulk consensus genome.

${ }^{\mathrm{C}} \mathrm{A}$ period on the haplotype indicates that the position carries the consensus base. SNVs are represented by the identity of the variant base. 
silent substitutions (Table S4 in Additional file 1). Among the non-synonymous variants, seven are nonsense mutations (one in the open reading frame (ORF) for the HBV $\mathrm{C}$ protein, one in the ORF for $\mathrm{X}$ protein, and five in the ORF for $\mathrm{S}$ protein) and one is a mutation in the stop-codon of the $\mathrm{C}$ gene that extends the ORF by six amino acids (Table S4 in Additional file 1); most of these mutations have been previously described [42-44] or exist in sequences from GenBank. Five of the nonsense mutations are located near the end of their ORFs and consequently may reduce or alter the expression or activity of the expressed proteins rather than abolishing expression altogether.

We identified 236 unique haplotypes from among the 9,072 haplotypes assembled from S7.1 that had a 100\% call rate across all SNV positions; these haplotypes were detected at frequencies ranging from $0.01 \%$ to $8.3 \%$. The actual number of unique haplotypes in the sample is likely to be higher because the library was not sequenced to saturation (Table 3) and any true SNVs present below the baseline error frequency cutoff were not included in the analysis (Table 1). A phylogenetic analysis revealed the existence of at least six distinct clades (Figure 3). Each clade consisted of at least one haplotype sequence that occurred at relatively high frequency and whose sequence is close to the common ancestor of the clade (for example, haplotype 1 in clade 2 and haplotype 5 in clade 4 ). Some clades have relatively deep branching patterns, which may indicate that they are evolving faster (for example, clade 6). Notably, five out of six clades contained at least one amino acid mutation that is likely to confer a fitness advantage (Figure 3). For instance, haplotypes in clade 4 contain one or more of the following mutations: nonsense mutations (sS235-stop and sW248-stop; refer to Table S4 in Additional file 1 for an explanation of residue numbering) that yield truncated surface proteins that are missing the 'a' determinant, i.e., the immunodominant region of HBsAg [45], and a mutation that results in immune escape $[46,47]$ (sP294T, usually referred to as sP120T, with respect to the small $\mathrm{S}$ protein). Also, another immune escape mutation, sQ303R [46,47] (usually referred to as sQ129R), co-exists on the same haplotype with sP294T in clades 1, 2 and 6 and comprises approximately 50\% of all haplotypes in the quasispecies. Finally, the sL360H mutation found in clade 5 has been predicted to disrupt homo-dimerization of the $S$ protein [48]. Taken together, the phylogenetic structure of viral quasispecies in this patient is consistent with a scenario where common haplotypes in the founder $\mathrm{HBV}$ population gave rise to sub-populations that evolved adaptive mutations required for viral persistence.

Intriguingly, a nonsense mutation (cQ181-stop) and a stop codon mutation (c-stop183QYSLDT) in the $C$ gene are both associated with clade 2 (Figure 3), and co-exist on approximately $93 \%$ of haplotypes that carry a mutation at either position in the quasispecies (Table S5 in Additional file 1). Furthermore, a closer inspection of all haplotypes among the S7.1 quasispecies shows that both immune escape mutations described above - sP294T and sQ303R are found on approximately $98 \%$ of haplotypes that contain both cQ181-stop and c-stop183QYSLDT but are only found on approximately $40 \%$ of haplotypes that are wild type at cQ181 and c-stop183 (Table S5 in Additional file 1). The co-occurrence of these four mutations on the same haplotypes strongly suggests that cQ181-stop and c-stop183QYSLDT may have arisen on an ancestral genome that already carried the sP294T and sQ303R mutations. A plausible scenario is that cQ181-stop, which removes the last two amino acids from the core protein, may have arisen as an intragenic suppressor of c-stop183QYSLDT to restore the fitness advantage conferred on this haplotype by the immune escape mutations. Importantly, these mutations are separated by $>1.3 \mathrm{~kb}$ and their occurrence with respect to one another cannot be resolved without long-range haplotype information.

\section{Future applications and possible improvements to BAsE-Seq} The current manifestation of BAsE-Seq contains a region of approximately $60 \mathrm{bp}$ at each end of the HBV genome where potential SNVs are missed because per-base coverage is significantly lower. This can be resolved by modifying the exonuclease digestion step, such as having additional time points, to allow these regions to be included in library preparation. With some modifications to the protocol that will involve the design of new primer sets, we anticipate that BAsE-Seq can be used to study other DNA viruses (for example, human papillomavirus) or low complexity but genetically heterogeneous regions (for example, B-cell or T-cell receptor sequences). However, further optimization of several steps in the BAsE-Seq protocol - genome amplification, exonuclease digestion and circularization steps - may be required to achieve longer haplotype sequences $(>4 \mathrm{~kb})$. By modifying the protocol to attach a barcode during reverse transcription, BAsE-Seq can also be applied to study RNA viruses (for example, HIV-1), or used to estimate the relative abundance of transcript isoforms. Taking into account the technical constraints in performing reverse transcription efficiently across long RNA templates, the future application of BAsE-Seq may be restricted to studying subgenomic regions of large RNA viruses. Finally, given the input requirements of BAsE-Seq $\left(10^{6} \mathrm{HBV}\right.$ genomes $)$, improvements to the efficiency of barcode assignment and genome amplification will be necessary in order to study clinical samples with low viral load.

\section{Alternative approaches to BAsE-Seq}

Recently, two methods using strategies similar to BAsESeq were published. The first method - called Tile-Seq - 


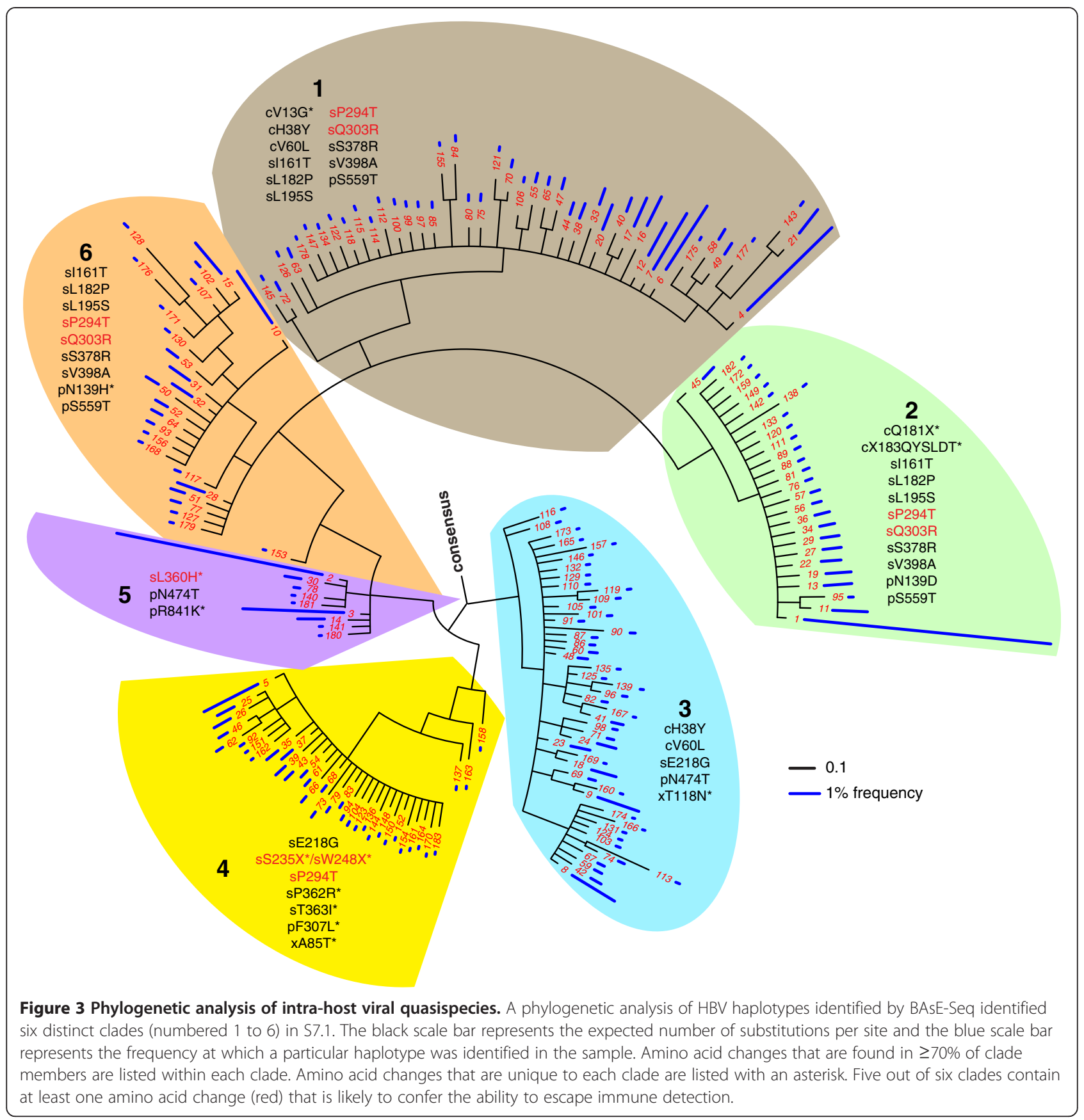

generated reads up to $3 \mathrm{~kb}$; however, this method did not produce haplotype information because individual template molecules were not tagged separately for downstream analysis [49]. Wu et al. [50] developed a method that obtained approximately $1.3 \mathrm{~kb}$ viral haplotypes, but relied on performing multiple nested PCRs to generate defined deletions of the initial template; this approach required a priori knowledge of the entire template sequence and its specificity in haplotype reconstruction was not demonstrated, thus raising concerns over the presence of chimeric sequences. New computational tools based on haplotype inference of viral quasispecies were recently released and used successfully to construct haplotypes from HIV-1 and hepatitis $C$ virus populations [51,52]; it would be of interest to evaluate their performance on an empirical dataset as we have shown here.

\section{Conclusions}

We have demonstrated that BAsE-Seq successfully performs single virion sequencing on HBV by generating viral haplotypes longer than $3 \mathrm{~kb}$, with substantially improved accuracy in SNV calling compared with conventional deep 
sequencing. A main advantage of BAsE-Seq over existing computational or molecular-based methods to obtain viral haplotypes is its high sensitivity and specificity. In a mixed HBV clone sample, BAsE-Seq accurately assembled haplotypes present at $\geq 0.4 \%$ frequency and achieved greater than $99.9 \%$ specificity. In a clinical sample, a sub-genomic haplotype present at approximately $0.08 \%$ frequency was validated by clonal sequencing. Notably, we used BAsESeq to obtain the first-time measurement of $>9,000$ viral haplotypes in a clinical sample, which allowed us to evaluate the intra-host population genetic structure of viral quasispecies in a chronic infection and track the cooccurrence of mutations located several kilobases apart across hundreds of unique haplotypes. The method described here is a significant improvement over existing methods to characterize viral quasispecies and will provide a useful tool to study the population genetic basis of viral persistence in a wide range of infections.

\section{Materials and methods Ethics statement}

All patients provided written informed consent according to the Declaration of Helsinki, and the study protocols were approved by the institutional review board of the participating hospitals.

\section{Hepatitis B virus clones}

Viral DNA from a chronic hepatitis B patient was isolated from $100 \mu \mathrm{l}$ of serum using the QIAamp UltraSens Virus kit (Qiagen, Venlo, Limburg, Netherlands). Fulllength HBV amplicons were obtained by PCR amplification of $5 \mathrm{ng}$ of viral DNA using previously published primers [53], gel-purified using a MinElute Gel Extraction kit (Qiagen), cloned into a pCR2.1-TOPO vector (Life Technologies, Carlsbad, CA, USA), and transformed into Escherichia coli ABLE K competent cells (Agilent, Santa Clara, CA, USA) following the manufacturers' protocols. Purified clones were verified for the presence of an approximately $3.2 \mathrm{~kb}$ insert by PCR, and full-length sequencing of the insert was performed using a primer walking approach (Table S1 in Additional file 1). The sequencing reactions were performed using a BIGDYE Terminator v3.1 kit (Life Technologies) and loaded on a 3730xl instrument (Life Technologies) for analysis. For BAsE-Seq library preparation, each HBV clone (Clone-1 and Clone-2) was linearized by restriction digest with NotI (NEB, Ipswich, MA, USA), gel-purified using a MinElute Gel Extraction kit (Qiagen), quantified using a Qubit dsDNA BR assay kit (Life Technologies), and diluted to $10^{6}$ copies $/ \mu$ l.

\section{Patient sample S7.1}

S7.1 is a genotype B HBV sample that was isolated from a chronic hepatitis B patient in 1990, and was selected from a database of samples in which clonal sequencing of the precore/core region had been previously described [54]. Briefly, viral DNA was isolated from $200 \mu \mathrm{l}$ of serum using the QIAamp DNA Blood Mini kit (Qiagen) and nested PCR amplification was carried out on the precore/core region. The 700-bp nested PCR product was purified, cloned into a pGEM-T vector (Promega, Madison, WI, USA), and transformed in E. coli JM109 cells (Promega). Positive clones were sequenced using vector-specific primers with BIGDYE Terminator on the 3730xl sequencer (Life Technologies). After quality trimming, a 466-bp region was obtained for each clone for further analysis. Viral DNA from S7.1 was also used for Deep-Seq and BAsE-Seq library preparation. To quantify the number of full-length genomes in the sample, real-time PCR was performed using the EXPRESS SYBR GreenER qPCR Supermix (Life Technologies) with primers (5'-ACTGTT CAAGCCTCCAAGCTG-3' and 5'-AAAAGTTGCATG GTGCTGGTGA-3') that amplified full-length amplicons of the HBV genome. The sample was measured in triplicate and its concentration was estimated by plotting the $\mathrm{C}_{\mathrm{t}}$ values against a standard curve that was generated using a 10-fold dilution series of HBV Clone-2. $10^{6} \mathrm{HBV}$ genomes from the sample were used for BAsE-Seq library preparation.

\section{BAsE-Seq library preparation}

A detailed protocol, including oligonucleotide sequences, is provided as Additional file 2. Using HBV-specific primers that contain universal sequence on their $5^{\prime}$ ends, $10^{6} \mathrm{HBV}$ genomes were uniquely assigned to a molecular barcode (20 random nucleotides) by performing two cycles of PCR using the Long PCR Enzyme Mix (Thermo Scientific, Waltham, MA, USA). Excess primers were removed by Exonuclease I (Enzymatics, Beverly, MA, USA). We clonally amplified 40,000 copies of barcode-tagged genomes using the Long PCR Enzyme Mix (Thermo Scientific) and universal primers, then digested with SbfI (NEB) to protect the barcode-proximal end from exonuclease digest. Next, unidirectional nested deletions from the barcode-distal end were generated using a combination of Exonuclease III and S1 Nuclease (Promega) to achieve a broad size distribution of fragments ranging from approximately $300 \mathrm{bp}$ to $3,200 \mathrm{bp}$. Barcode-containing fragments were purified using streptavidin-coated Dynabeads (Life Technologies) and subjected to end repair using T4 DNA polymerase and T4 Polynucleotide Kinase (NEB). End-repaired molecules were circularized by intramolecular ligation using T4 DNA Ligase (NEB) and uncircularized molecules were removed by digestion with Lambda Exonuclease and Exonuclease I (Enzymatics). After circularization, different regions from each viral genome were juxtaposed with the barcode assigned to that genome. The circularized molecules were used as a template for random fragmentation and adapter tagging using the Nextera XT kit 
(Illumina, San Diego, CA, USA). During PCR enrichment, a set of custom primers was used to randomly incorporate the 'P5' adapter and place the 'P7' adapter next to the barcode. Each library was subjected to size selection to remove fragments $<400$ bp using AMPure XP beads (Beckman Coulter, Brea, CA, USA), verified on a Bioanalyzer (Agilent), and quantified by real-time PCR using a Library Quantification kit (KAPA Biosystems, Wilmington, MA, USA). We loaded $18 \mathrm{pM}$ of each library on a Miseq 300cycle reagent cartridge (Illumina) for $2 \times 150$ bp sequencing. During sequencing, a custom sequencing primer that annealed to the forward priming sequence of HBV was used for the second read to ensure that it begins at the barcode.

During library preparation for sample S7.1, barcodes were separately assigned to patient-specific viral genomes and HBV Clone-2 (which we refer to as an 'internal standard'). The barcodes assigned to the internal standard contain a two-base insertion that served to distinguish them from patient-specific barcodes. After barcoding, 5,000 copies of the internal standard were mixed with 35,000 copies of patient-specific genomes and used to build a BAsE-Seq library.

\section{BAsE-Seq data analysis}

Read pairs were trimmed to remove the barcode, universal and adapter sequences using Fastx (v0.0.13) and Trimmomatic (v0.30). After trimming, read pairs that were $\geq 15 \mathrm{bp}$ in length were aligned to a 'bulk consensus' genome using default parameters on the Burrows-Wheeler Aligner (BWA; v0.6.1). The bulk consensus genome used to analyze the mixed-clone libraries was the Clone-2 reference sequence obtained by Sanger sequencing. For patient sample S7.1, the bulk consensus genome was obtained by first aligning reads using BWA against a genotype B HBV reference sequence (GenBank accession number AF121245.1) and extracting the major base call (or indel) at each position. Then, reads were aligned to the newly derived bulk consensus genome and the process was repeated iteratively until saturation was achieved with the proportion of mapped reads. BAsE-Seq primers amplify the HBV genomic region spanning base positions 42 to 3,156 and 39 to 3,219 on the Clone- 2 and S7.1 consensus genomes, respectively.

For 'bulk' data analysis, BAM files from concordantly aligned reads (read 1: forward strand; read 2: reverse strand) were used as input for variant calling using the -Q25 setting (ignore non-reference bases $<$ Q25) in LoFreq [40] (v2.0.0). For 'individual' genome analysis, concordantly aligned reads were de-multiplexed into individual genome BAM files using the identity of the barcode associated with each read pair. PCR duplicates associated with individual genomes were removed using the rmdup function in samtools (v0.1.18) [55]. Next, vcf files were generated using the mpileup (-C50 -Q25 -d 1000 -DEu) function in samtools (v0.1.18). An 'individual consensus' sequence was obtained for each viral genome by extracting the consensus base call at each position. Specifically, a consensus base call was generated if the per-base coverage was $\geq 4$ reads and the concordance was $\geq 0.7$, that is, the major base call occurred in at least $70 \%$ of reads. The number of 'high quality genomes' extracted from each sample will depend on the genome coverage threshold (Table S3 in Additional file 1). We used a higher genome coverage threshold (85\%) for the mixed clone libraries to maximize the number of haplotypes with complete SNV calls and a lower genome coverage threshold $(50 \%)$ for the patient sample to maximize the number of haplotypes recovered for downstream analysis. SNVs - relative to the bulk consensus genome - were identified from individual viral genomes and filtered for those that occurred in $\geq 2$ genomes in the sample and where base calls were generated in $\geq 1,500$ genomes at that position. Finally, haplotypes were constructed for each library using the filtered SNVs.

To determine the library-specific error rate for S7.1, read pairs associated with the internal standard were analyzed separately from patient-specific reads. Errors in the internal standard - SNVs relative to the Clone-2 sequence were identified from individual viral genomes and filtered for those where base calls were generated in $\geq 100$ genomes at that position. The highest per-base error observed in the data was used to set the baseline error frequency for SNVs observed in the patient sample. We defined this as the frequency threshold below which the SNV might be due to an error and above which the SNV is treated as a true SNV.

To maximize the recovery of haplotype information, we implemented a method to impute the identity of ambiguous bases (Ns) in haplotypes that may arise as a result of low per-base coverage or concordance. First, information was shared across haplotypes using a conservative clustering approach. Haplotypes were clustered using a greedy algorithm that sequentially builds a set of seeds (for clusters) considering haplotypes in order of decreasing abundance. Haplotypes that perfectly matched one of the existing seeds (ignoring Ns) were clustered with them and used to generate a consensus haplotype. Consensus bases were determined by taking the most common base if its frequency was at least $10 \%$ greater than the next most common base and using an $\mathrm{N}$ otherwise. Haplotypes with Ns in more than $10 \%$ of their bases and less than two non-reference bases were excluded from consideration as potential seeds and were reported as singleton clusters.

Unless otherwise stated, custom perl and shell scripts were used for data analysis and are available upon request. 


\section{Phylogenetic analysis}

Haplotypes with less than 10 Ns and count $\geq 10$ were used to construct a phylogenetic tree using MrBayes [56] (version 3.2.2; consensus based on 10,000 sample trees, GTR model, $\gamma$-distributed rate variation, burn-in of 100,000 iterations and sampling every 200 iterations) and plotted in $\mathrm{R}$ using the Ape package [57].

\section{Deep-Seq library preparation}

HBV-specific primers (5'-GCTCTTCTTTTTCACCTCT GCCTAATCA-3' and 5'-GCTCTTCAAAAAGTTGCA TGGTGCTGG-3') were used to generate a full-length amplicon of the HBV genome using the PfuUltra II Fusion HS DNA Polymerase (Agilent) according to the manufacturer's instructions. The PCR product was run on a $1 \%$ agarose gel and the approximately $3.2 \mathrm{~kb}$ fragment was purified using the QIAquick Gel Extraction Kit (Qiagen). The purified sample was sheared into 100 to $300 \mathrm{bp}$ fragments using the following conditions on the Covaris S2 (Covaris, Woburn, MA, USA): duty cycle, 20\%; intensity, 5; cycles per burst, 200; time, 110 seconds. The fragments were purified using the QIAquick PCR purification kit (Qiagen) and a Deep-Seq library was prepared using the KAPA Library Preparation Kit (KAPA Biosystems) following the manufacturer's instructions. TruSeq adapters and dual-indexing primers (Illumina) were used for library preparation and the final PCR step was performed using PfuUltra II Fusion HS DNA Polymerase according to the manufacturer's instructions. The library was quantified by real-time PCR using a Library Quantification kit (KAPA Biosystems) and loaded on a flowcell for $2 \times 101 \mathrm{bp}$ sequencing on a HiSeq 2500 (Illumina).

\section{Deep-Seq data analysis}

The essential steps in our data analysis pipeline follow the protocol described in Aw et al. [39]. In brief, a consensus sequence for the sample was obtained by iterative alignment of the sequence reads against a reference using BWA (as described above). After the final mapping, SNVs were identified using LoFreq [40] (v2.0.0) and variants within the primer region (base positions 1 to 21 and 3201 to 3220 ) were removed.

\section{Data availability}

Raw data have been deposited in the NCBI Sequence Read Archive under accession number PRJNA251790.

\section{Additional files}

Additional file 1: Figure S1. Barcode assignment and error removal. Figure S2. Allele frequency in mixed-clone libraries Figure S3. Coverage depth for individual genomes. Figure S4. Per-base coverage in S7.1 using Deep-Seq. Table S1. Primers used for sequencing HBV clones. Table S2.
SNPs between Clone-1 and Clone-2. Table S3. Genome coverage per sample by BAsE-Seq. Table S4. True SNVs identified by BAsE-Seq in S7.1. Table S5. Haplotype analyses of immune escape mutations.

Additional file 2: BAsE-Seq detailed protocol.

\section{Abbreviations}

BAsE-Seq: Barcode-directed Assembly for Extra-long Sequences; bp: base pair; BWA: Burrows-Wheeler Aligner; HBV: hepatitis B virus; MAF: minor allele frequency; ORF: open reading frame; PCR: polymerase chain reaction; SNP: single nucleotide polymorphism; SNV: single nucleotide variant.

\section{Competing interests}

LZH, WFB and SRQ are co-inventors on a patent application relating to the results herein. The other co-authors declare no competing financial interests.

\section{Authors' contributions}

SRQ, WFB and LZH conceived BAsE-Seq. LZH, SH and HTW developed BAsE-Seq. LZH and HTW performed data analysis for BAsE-Seq. NN performed haplotype imputation and phylogenetic analysis. PPKA, AW, PFS, LZH, NN and MLH generated and analyzed Deep-Seq data, and evaluated its performance against BAsE-Seq. YC and SGL generated clonal sequencing data. $\mathrm{LZH}$, WFB and SRQ wrote the draft manuscript and all authors contributed to the final manuscript. All authors read and approved the final manuscript.

\section{Acknowledgements}

We thank Polly Poon for generating sequencing data and Sarah $\mathrm{Ng}$ for critical reading of the manuscript. We also thank Antonio Bertoletti for providing a serum sample from a clinical trial conducted by Hoffmann-La Roche (NCT00962871). This work was supported by a Visiting Investigator Programme grant (to SRQ; project numbers 0921100080 and 1235e00049) and a Career Development Award (to LZH; project number JCO13302FG059) from the Joint Council Office of the Agency for Science, Technology and Research in Singapore.

\section{Author details}

${ }^{1}$ Institute of Molecular and Cell Biology, Agency for Science, Technology and Research, Singapore 138673, Singapore. ${ }^{2}$ Genome Institute of Singapore, Agency for Science, Technology and Research, Singapore 138672, Singapore. ${ }^{3}$ Department of Medicine, Yong Loo Lin School of Medicine, National University of Singapore, Singapore 117597, Singapore. ${ }^{4}$ Department of Gastroenterology and Hepatology, National University Health System, Singapore 119074, Singapore. ${ }^{5}$ Department of Pathogen Biology, London School of Hygiene and Tropical Medicine, London WC1E 7HT, UK

${ }^{6}$ Departments of Bioengineering and Applied Physics, Stanford University and Howard Hughes Medical Institute, Stanford, CA 94305, USA. Visting Investigator, Institute of Molecular and Cell Biology, Agency for Science, Technology and Research, Singapore 138673, Singapore. ${ }^{8}$ Present address: Molecular Biomarkers \& Diagnostics, Translational Medicine Research Centre Singapore, Merck Sharp \& Dohme, Singapore 138665, Singapore. ${ }^{9}$ Present address: Chugai Pharmabody Research Pte Ltd, Singapore 138623, Singapore.

Received: 7 October 2014 Accepted: 30 October 2014

Published online: 19 November 2014

\section{References}

1. Domingo E, Sabo D, Taniguchi T, Weissmann C: Nucleotide sequence heterogeneity of an RNA phage population. Cell 1978, 13:735-744.

2. Duffy $S$, Shackelton LA, Holmes E: Rates of evolutionary change in viruses: patterns and determinants. Nat Rev Genet 2008, 9:267-276.

3. Burch $\mathrm{CL}$, Chao L: Evolvability of an RNA virus is determined by its mutational neighbourhood. Nature 2000, 406:625-628.

4. Domingo E, Sheldon J, Perales C: Viral quasispecies evolution. Microbiol Mol Biol Rev 2012, 76:159-216.

5. Eigen M: Viral quasispecies. Sci Am 1993, 269:42-49.

6. Lauring A, Andino R: Quasispecies theory and the behavior of RNA viruses. PLoS Pathog 2010, 6:e1001005.

7. Henn MR, Boutwell CL, Charlebois P, Lennon NJ, Power KA, Macalalad AR, Berlin AM, Malboeuf CM, Ryan EM, Gnerre S, Zody MC, Erlich RL, Green LM, Berical A, Wang Y, Casali M, Streeck H, Bloom AK, Dudek T, Tully D, Newman R, Axten KL, Gladden AD, Battis L, Kemper M, Zeng Q, Shea TP, Gujja S, Zedlack C, 
Gasser $\mathrm{O}$, et al: Whole genome deep sequencing of HIV-1 reveals the impact of early minor variants upon immune recognition during acute infection. PLOS Pathog 2012, 8:e1002529.

8. Margeridon-Thermet $S$, Shulman NS, Ahmed A, Shahriar R, Liu T, Wang C, Holmes SP, Babrzadeh F, Gharizadeh B, Hanczaruk B, Simen BB, Egholm M, Shafer RW: Ultra-deep pyrosequencing of hepatitis $B$ virus quasispecies from nucleoside and nucleotide reverse-transcriptase inhibitor (NRTI)-treated patients and NRTI-naive patients. J Infect Dis 2009, 199:1275-1285.

9. Simen BB, Simons JF, Hullsiek KH, Novak RM, Macarthur RD, Baxter JD, Huang C, Lubeski C, Turenchalk GS, Braverman MS, Desany B, Rothberg JM, Egholm M, Kozal MJ, AIDS TBCPfCRo: Low-abundance drug-resistant viral variants in chronically HIV-infected, antiretroviral treatment-naive patients significantly impact treatment outcomes. J Infect Dis 2009, 199:693-701.

10. Solmone M, Vincenti D, Prosperi MC, Bruselles A, Ippolito G, Capobianchi MR: Use of massively parallel ultradeep pyrosequencing to characterize the genetic diversity of hepatitis B virus in drug-resistant and drug-naive patients and to detect minor variants in reverse transcriptase and hepatitis B S antigen. J Virol 2009, 83:1718-1726.

11. Acevedo A, Brodsky $L$, Andino R: Mutational and fitness landscapes of an RNA virus revealed through population sequencing. Nature 2014, 505:686-690.

12. Jabara CB, Jones CD, Roach J, Anderson JA, Swanstrom R: Accurate sampling and deep sequencing of the HIV-1 protease gene using a Primer ID. Proc Natl Acad Sci U S A 2011, 108:20166-20171.

13. Kinde I, Wu J, Papadopoulos N, Kinzler KW, Vogelstein B: Detection and quantification of rare mutations with massively parallel sequencing. Proc Natl Acad Sci U S A 2011, 108:9530-9535.

14. Mardis ER: Next-generation sequencing platforms. Annu Rev Anal Chem 2013, 6:287-303.

15. Prosperi MC, Yin L, Nolan DJ, Lowe AD, Goodenow MM, Salemi M: Empirical validation of viral quasispecies assembly algorithms: state-of-the-art and challenges. Sci Rep 2013, 3:2837

16. Eid J, Fehr A, Gray J, Luong K, Lyle J, Otto G, Peluso P, Rank D, Baybayan P, Bettman B, Bibillo A, Bjornson K, Chaudhuri B, Christians F, Cicero R, Clark S, Dalal R, Dewinter A, Dixon J, Foquet M, Gaertner A, Hardenbol P, Heiner C, Hester K, Holden D, Kearns G, Kong X, Kuse R, Lacroix Y, Lin S, et al: Real-time DNA sequencing from single polymerase molecules. Science 2009, 323:133-138.

17. Travers KJ, Chin CS, Rank DR, Eid JS, Turner SW: A flexible and efficient template format for circular consensus sequencing and SNP detection. Nucleic Acids Res 2010, 38:e159.

18. Branton D, Deamer D, Marziali A, Bayley H, Benner S, Butler T, Di Ventra M, Garaj S, Hibbs A, Huang $X$, Jovanovich S, Krstic P, Lindsay S, Ling $X$, Mastrangelo C, Meller A, Oliver J, Pershin Y, Ramsey J, Riehn R, Soni G, Tabard-Cossa V, Wanunu M, Wiggin M, Schloss J: The potential and challenges of nanopore sequencing. Nat Biotechnol 2008, 26:1146-1153.

19. Maitra R, Kim J, Dunbar W: Recent advances in nanopore sequencing. Electrophoresis 2012, 33:3418-3428.

20. Hiatt JB, Patwardhan RP, Turner EH, Lee C, Shendure J: Parallel, tag-directed assembly of locally derived short sequence reads. Nat Methods 2010, 7:119-122.

21. Schwartz JJ, Lee C, Hiatt JB, Adey A, Shendure J: Capturing native long-range contiguity by in situ library construction and optical sequencing. Proc Natl Acad Sci U S A 2012, 109:18749-18754.

22. Kim H, Jee $Y$, Song B, Hyun J, Mun H, Kim H, Oh E, Yoon J, Kim Y, Lee H, Hwang $E$, Cha C, Kook Y, Kim B: Analysis of hepatitis B virus quasispecies distribution in a Korean chronic patient based on the full genome sequences. J Med Virol 2007, 79:212-219.

23. Li H, Stoddard M, Wang S, Blair L, Giorgi E, Parrish E, Learn G, Hraber P, Goepfert P, Saag M, Denny T, Haynes B, Hahn B, Ribeiro R, Perelson A, Korber B, Bhattacharya T, Shaw G: Elucidation of Hepatitis C virus transmission and early diversification by single genome sequencing. PLoS Pathog 2012, 8:e1002880.

24. Palmer S, Kearney M, Maldarelli F, Halvas EK, Bixby CJ, Bazmi H, Rock D, Falloon J, Davey RT, Dewar RL, Metcalf JA, Hammer S, Mellors JW, Coffin JM: Multiple, linked human immunodeficiency virus type 1 drug resistance mutations in treatment-experienced patients are missed by standard genotype analysis. J Clin Microbiol 2005, 43:406-413.

25. Salazar-Gonzalez JF, Salazar MG, Keele BR, Learn GH, Giorgi EE, Li H, Decker JM, Wang S, Baalwa J, Kraus MH, Parrish NF, Shaw KS, Guffey MB, Bar KJ, Davis KL,
Ochsenbauer-Jambor C, Kappes JC, Saag MS, Cohen MS, Mulenga J, Derdeyn CA, Allen S, Hunter E, Markowitz M, Hraber P, Perelson AS, Bhattacharya T, Haynes BF, Korber BT, Hahn BH, et al: Genetic identity, biological phenotype, and evolutionary pathways of transmitted/ founder viruses in acute and early HIV-1 infection. J Exp Med 2009, 206:1273-1289.

26. Wang HY, Chien MH, Huang HP, Chang HC, Wu CC, Chen PJ, Chang MH, Chen DS: Distinct hepatitis B virus dynamics in the immunotolerant and early immunoclearance phases. J Virol 2010, 84:3454-3463.

27. Fan HC, Wang J, Potanina A, Quake SR: Whole-genome molecular haplotyping of single cells. Nat Biotechnol 2010, 29:51-57.

28. Kaper F, Swamy S, Klotzle B, Munchel S, Cottrell J, Bibikova M, Chuang H, Kruglyak S, Ronaghi M, Eberle M, Fan J: Whole-genome haplotyping by dilution, amplification, and sequencing. Proc Natl Acad Sci U S A 2013, 110:5552-5557.

29. Peters BA, Kermani BG, Sparks AB, Alferov O, Hong P, Alexeev A, Jiang Y, Dahl F, Tang YT, Haas J, Robasky K, Zaranek AW, Lee J, Ball MP, Peterson JE, Perazich H, Yeung G, Liu J, Chen L, Kennemer MI, Pothuraju K, Konvicka K, Tsoupko-Sitnikov M, Pant KP, Ebert JC, Nilsen GB, Baccash J, Halpern AL, Church GM, Drmanac R: Accurate whole-genome sequencing and haplotyping from 10 to 20 human cells. Nature 2012, 487:190-195.

30. Voskoboynik A, Neff NF, Sahoo D, Newman AM, Pushkarev D, Koh W, Passarelli B, Fan HC, Mantalas GL, Palmeri KJ, Ishizuka KJ, Gissi C, Griggio F, Ben-Shlomo R, Corey DM, Penland L, White RA, Weissman IL, Quake SR: The genome sequence of the colonial chordate. Botryllus schlosseri. elife 2013, 2:e00569.

31. Beck J, Nassal M: Hepatitis B virus replication. World J Gastroenterol 2007, 13:48-64.

32. Nowak MA, Bonhoeffer S, Hill AM, Boehme R, Thomas HC, McDade H: Viral dynamics in hepatitis B virus infection. Proc Natl Acad Sci U S A 1996, 93:4398-4402

33. Osiowy C, Giles E, Tanaka Y, Mizokami M, Minuk GY: Molecular evolution of hepatitis B virus over 25 years. J Virol 2006, 80:10307-10314.

34. Vollmers C, Sit RV, Weinstein JA, Dekker CL, Quake SR: Genetic measurement of memory B-cell recall using antibody repertoire sequencing. Proc Natl Acad Sci U S A 2013, 110:13463-13468.

35. Henikoff S: Unidirectional digestion with exonuclease III creates targeted breakpoints for DNA sequencing. Gene 1984, 28:351-359.

36. Adey A, Morrison HG, Asan Xun X, Kitzman JO, Turner EH, Stackhouse B, Mackenzie AP, Caruccio NC, Zhang X, Shendure J: Rapid, low-input, low-bias construction of shotgun fragment libraries by high-density in vitro transposition. Genome Biol 2010, 11:R119.

37. Qiu X, Wu L, Huang H, McDonel PE, Palumbo AV, Tiedje JM, Zhou J: Evaluation of PCR-generated chimeras, mutations, and heteroduplexes with 16S rRNA gene-based cloning. Appl Environ Microbiol 2001, 67:880-887.

38. Thompson JR, Marcelino LA, Polz MF: Heteroduplexes in mixed-template amplifications: formation, consequence and elimination by 'reconditioning PCR'. Nucleic Acids Res 2002, 30:2083-2088.

39. Aw PP, De Sessions P, Wilm A, Hoang L, Nagarajan N, Sessions O, Hibberd ML: Next-generation whole genome sequencing of dengue virus. Methods $\mathrm{Mol}$ Biol 2014, 1138:175-195.

40. Wilm A, Aw PP, Bertrand D, Yeo GH, Ong SH, Wong CH, Khor CC, Petric R, Hibberd ML, Nagarajan N: LoFreq: a sequence-quality aware, ultra-sensitive variant caller for uncovering cell-population heterogeneity from high-throughput sequencing datasets. Nucleic Acids Res 2012, 40:11189-11201.

41. Drake JW, Charlesworth B, Charlesworth D, Crow JF: Rates of spontaneous mutation. Genetics 1998, 148:1667-1686.

42. Grabarczyk P, Garmiri P, Liszewski G, Doucet D, Sulkowska E, Brojer E, Allain JP, Group PBTCVS: Molecular and serological characterization of hepatitis B virus genotype A and D infected blood donors in Poland. J Viral Hepat 2010, 17:444-452.

43. León B, Taylor L, Vargas M, Luftig RB, Albertazzi F, Herrero L, Visona K: HBx M130K and V131I (T-A) mutations in HBV genotype F during a follow-up study in chronic carriers. Virol J 2005, 2:60.

44. Shen T, Yan XM, Zhang JP, Wang JL, Zuo RX, Li L, Wang LP: Evolution of Hepatitis B virus in a chronic HBV-infected patient over 2 years. Hepat Res Treat 2011, 2011:939148.

45. Echevarría J, Avellón A: Hepatitis B virus genetic diversity. J Med Virol 2006, 78:S36-S42 
46. Huang C, Yuan Q, Chen PJ, Zhang Y, Chen C, Zheng Q, Yeh S, Yu H, Xue Y, Chen Y, Liu P, Ge S, Zhang J, Xia N: Influence of mutations in hepatitis B virus surface protein on viral antigenicity and phenotype in occult HBV strains from blood donors. J Hepatol 2012, 57:720-729.

47. Kwei K, Tang X, Lok AS, Sureau C, Garcia T, Li J, Wands J, Tong S: Impaired virion secretion by hepatitis $B$ virus immune escape mutants and its rescue by wild-type envelope proteins or a second-site mutation. $J$ Virol 2013, 87:2352-2357.

48. van Hemert FJ, Zaaijer HL, Berkhout B, Lukashov W: Mosaic amino acid conservation in 3D-structures of surface protein and polymerase of hepatitis B virus. Virology 2008, 370:362-372.

49. Lundin S, Gruselius J, Nystedt B, Lexow P, Käller M, Lundeberg J: Hierarchical molecular tagging to resolve long continuous sequences by massively parallel sequencing. Sci Rep 2013, 3:1186.

50. Wu NC, De La Cruz J, Al-Mawsawi LQ, Olson CA, Qi H, Luan HH, Nguyen N, Du Y, Le S, Wu TT, Li X, Lewis MJ, Yang OO, Sun R: HIV-1 quasispecies delineation by tag linkage deep sequencing. PLoS One 2014, 9:e97505.

51. Töpfer A, Zagordi O, Prabhakaran S, Roth V, Halperin E, Beerenwinkel N: Probabilistic inference of viral quasispecies subject to recombination. J Comput Biol 2013, 20:113-123.

52. Töpfer A, Marschall T, Bull RA, Luciani F, Schönhuth A, Beerenwinkel N: Viral quasispecies assembly via maximal clique enumeration. PLoS Comput Biol 2014, 10:e1003515.

53. Zhang Q, Wu G, Richards E, Jia S, Zeng C: Universal primers for HBV genome DNA amplification across subtypes: a case study for designing more effective viral primers. Virol J 2007, 4:92

54. Lim SG, Cheng Y, Guindon S, Seet BL, Lee LY, Hu P, Wasser S, Peter FJ, Tan T, Goode M, Rodrigo AG: Viral quasi-species evolution during hepatitis Be antigen seroconversion. Gastroenterology 2007, 133:951-958.

55. Li H, Handsaker B, Wysoker A, Fennell T, Ruan J, Homer N, Marth G, Abecasis G, Durbin R, 1000 Genome Project Data Processing Subgroup: The Sequence Alignment/Map format and SAMtools. Bioinformatics 2009, 25:2078-2079.

56. Ronquist F, Teslenko M, van der Mark P, Ayres DL, Darling A, Höhna S, Larget B, Liu L, Suchard MA, Huelsenbeck JP: MrBayes 3.2: efficient Bayesian phylogenetic inference and model choice across a large model space. Syst Biol 2012, 61:539-542.

57. Paradis E, Claude J, Strimmer K: APE: Analyses of phylogenetics and evolution in R language. Bioinformatics 2004, 20:289-290.

doi:10.1186/s13059-014-0517-9

Cite this article as: Hong et al:: BAsE-Seq: a method for obtaining long viral haplotypes from short sequence reads. Genome Biology 2014 15:517.

\section{Submit your next manuscript to BioMed Central and take full advantage of:}

- Convenient online submission

- Thorough peer review

- No space constraints or color figure charges

- Immediate publication on acceptance

- Inclusion in PubMed, CAS, Scopus and Google Scholar

- Research which is freely available for redistribution 